\title{
XXXI. Mittheilungen aus dem mineralogischen Institut der Universität Strassburg. 13.
}

\author{
Glaserit, Blödit, Kainit und Boracit von Douglashall \\ bei Westeregeln.
}

Von

H. Bücking in Strassburg.

(Mit Tafel X.)

\section{Glaserit.}

Zu Douglashall bei Westeregeln haben sich in den letzten Jabren in dem Astrakanit (Blödit), welcher mit Steinsalz verwachsen eine mehrfach unterbrochene Ablagerung von wechselnder Mächtigkeit zwischen Kainit und Steinsalz, in der Kieseritregion, bildet, Krystalle von schwefelsaurem Natrium-Kalium gefunden, deren Untersuchung mir durch die Freundlichkeit der Herren $\mathrm{Ochsen} \mathrm{i} \mathrm{u} \mathrm{in} \mathrm{Marburg} \mathrm{und} \mathrm{A.} \mathrm{Naupert} \mathrm{in}$ Douglashall ermöglicht wurde.

Die Krystalle, welche in ihrer grösslen Ausdehnung in der Regel etwa $5 \mathrm{~mm}$, in seltenen Fällen aber auch bis zu $20 \mathrm{~mm}$ messen, liegen einzeln, und dann ringsum ausgebildet, oder in Gruppen von mehreren, regellos mit einander verbundenen Individuen in dem Astrakanitgestein eingewachsen. Sie besitzen einen fettartigen Glasglanz, sind durchsichtig bis durchscheinend, theils farblos, theils grau und gelblich, zuweilen durch Einschlüsse von unregelmässig vertheillen schwarzen, kohligen Substanzen etwas dunkel oder durch fein vertheiltes Eisenoxyd röthlich gefärbt. Ihre Härte ist $2 \frac{3}{4}$ bis 3 . Das specifische Gewicht, an verschiedenen vollkommen homogen aussehenden Bröckchen in Methylenjodid bestimmt, schwankt im Allgemeinen zwischen 2,650 und 2,656; ein Krystall, der auf seine Homogenilät nicht weiter gepruft wurde, besass das specifische Gewicht 2,632. Diese Differenzen sind offenbar bedingt durch unregelmässig vertheille, 
kleine, etwa stecknadelkopf- bis fast erbsengrosse Einschlusse von Steinsalz, welche auf der Bruchfläche der Krystalle an ihrer guten Spalıbarkeit und an dem starken Glasglan\% leicht zu erkennen sind.

Die Mehrzahl der mir vorliegenden Krystalle ist deutlich rbomboëdris ch ausgebildet (Fig. 1, 2 und 3), einige dagegen haben das Ausseben einfacher rhombischer Krystalle (Hig. 4), während wieder andere eine grosse Aehnlichkeit mit den Durchkreuzungszwillingen von Aragonit besitzen und an die von G. vom R ath in Poggend. Ann. 1873, Ergänz.-Bd. 6, 359 etc. beschriebenen Arcanilkrystalle aus dem untermiocänen Steinsalz yon Racalmuto in Sicilien erinnern (Fig. 5 a und 5 b, 6 a und 6 b). Sowohl die Messungen, als insbesondere die optische Untersuchung auch derjenigen Krystalle, welche ihrer Form nach hätten rhombisch sein können, ergaben ubereinstimmend das Resultat, dass sämmiliche Krystalle, sowohl die einfachen, als die Aragonit-ähnlichen scheinbaren Drillinge, dem bexag o n a len Systeme zugehören.

Die rhomboëdrisch ausgebildeten Krystalle zeigen in der Regel vorwaltend ein flaches Rhomboëder $e$ und etwas kleiner entwickelt die Basis $c$ und das Prisma erster Ordnung $m$ (Fig. 1). Wird nach dem Vorgange von Mitscherlich (Pogg. Ano. 1843, ธ8, 468) das Rhomboëder, welches an dem bei der Aufarbeitung von Kelplauge erbaltenen "platten " schwefelsauren Natrium-Kalium randlich auftritt, als das primäre bezeichnet, so erhält jenes flache Rhomboëder $e$ das Zeichen $x\{10 \bar{T} 2\} \frac{1}{2} R$, wobei aber das Vorzeichen nicht näher bestimmt werden konnte. Es liegen ausser jenen häufiger auftretenden Krystallen auch einige vor, welche nur von $\frac{1}{2} R$ begrenzl sind, und ebenso Krystalle, welche nach $c=\{0001\} 0 R$ dicktafelartig sind und randlich entweder $e=\frac{1}{2} R$ oder $m=\{10 \bar{T} 0\} \infty R$ vorherrschend oder nur allein entwickelt zeigen (vergl. Fig. 2 und 3).

Selten sind die Krystalle so flächenreich, wie ein kleiner, nur $3 \mathrm{~mm}$ breiler und $1 \mathrm{~mm}$ dicker Krystall, welcher in seinem Habitus dem in Fig. 3 abgebildeten im Allgemeinen entspricht, aber ausser den grossentwickelten Formen $c, e$ und $m$ auch noch $e^{\prime}=x\{01 \bar{T} 2\}-\frac{1}{2} R, r=x\{10 \overline{1} 1\} R, r^{\prime}=$ $x\{01 T 1\}-R$ und $n=x\{11 \overline{2} 0\} \infty P 2$ erkennen lässt. Von $n$ sind allerdings nur zwei sehr schmale Flächen vorhanden. Nur einmal erscheint als winzige, matte, kein Reflexbild liefernde Abstumpfung einer Combinationskante von $c$ mit $e^{\prime}$ die an anderen Krystallen nicht beobachtete und daber immerhin nocb etwas problematische Form $g=x\{01 \overline{1} 4\}-\frac{1}{4} R$.

An diesem flächenreichen Krystalle (Nr. 2) sind die grösser entwickelten Flächen von $c, e$ und $m$ ausnahmsweise stark glänzend und gut spiegelnd; er wurde deshalb zur Bestimmung des Axenverhältnisses verwendet. Die anderen Krystalle, deren Flächen im Allgemeinen etwas raub sind, eignen sich weniger zu genauen Messungen. Zumal die breiteren unter ihnen sind nichl selten aus einer grösseren Zabl nicht ganz genau parallel orientirter 
kleinerer Individuen aufgebaut, deren Basen zu einer mehrfach gebrochenen oder unregelmässig gewölbten Fläche zusammentreten. Nur die Messungen, welche an einem $4 \mathrm{~mm}$ breilen Krystalle (Nr. 1) ausgefuhrt wurden, lieferlen bessere Werthe.

Es wurden uberhaupt folgende Winkel als Mittel aus mehreren Messungen bestimmt:

$$
\begin{aligned}
& \text { Gemessen: Berechnel : } \\
& \begin{array}{l}
\text { An Krystall Nr. 2: }(0001):\left(10 T^{2}\right)={ }^{*} 36^{0} 38^{\prime} \\
-\quad-\quad \text { Nr. 1: }(0001):(10 \overline{1} 2)=3638 \quad-
\end{array} \\
& \text { - } \quad \text { Nr. 1:(10T2):(T102) }=6219 \quad 6214 \\
& -\quad-\quad N r \cdot 2:(0001):(1011)=56 \quad 0 \quad 56 \quad 5 \\
& \text { - } \quad \text { Nr. 2: (0001):(01T4) = } 2024 \text { appr. } 1938
\end{aligned}
$$

Aus dem Winkel (0001):(10耳'2), für welchen an beiden Krystallen im Miltel vollkommen ubereinstimmende Werthe, zwischen den einzelnen Messungen aber nur geringe Schwankungen - am Krystall Nr. 2 zwischen den Grenzen $36^{\circ} 24^{\prime}$ und $36^{\circ} 44^{\prime}$, am Krystall Nr. 1 zwischen den Grenzen $36^{\circ} 24^{\prime}$ und $36^{\circ} 50^{\prime}$ - gefunden wurden, berechnel sich das Axenverhältniss

$$
a: c=1: 1,2879,
$$

welches nur wenig von dem aus Mitscherlich's Messung berechnelen Werthe abweicht, vor jenem aber wohl deshalb den Vorzug verdient, weil Mitscherlich's Messung bei der schlechten Ausbildung der von ihm untersuchten Krystalle nur zu einem angenäherten Werthe führen konnte.

Einige Krystalle erhalten ein abweichendes Aussehen dadurcb, dass sie von $+\frac{1}{2} R(e)$ und $-\frac{1}{2} R\left(e^{\prime}\right)$ jedesmal nur die beiden Flächen gross entwickelt zeigen, welche mit $0 R(c)$ zusammen in einer Zone liegen (Fig. 4). Die Krystalle erinnern dadurch an rhombische, nach der Brachydiagonale etwas verlängerte Krystalle, an welchen neben dem einem hexagonalen ähnlichen Prisma noch das Brachypinakoid und am Ende die Basis und ein Brachydoma erscheinen. Indessen erwiesen sich alle so ausgebildeten Krystalle bei näherer Untersuchung in allen ihren Theilen als deutlich optisch einaxig. Sie sind demnach als rhomboëdrische Combinationen der Formen $c, m, e$ und $e^{\prime}$ aufzufassen, wenn man nicht etwa wegen der grossen Aehnlichkeit der beiden Flächen, welche zusammen die brachydomatische Form bilden, an Durchkreuzungszwillinge rhomboëdrischer Krystalle der Combination $c, m, e\left(0 R, \infty R, \frac{1}{2} R\right)$ nach $0 R(c)$ oder an boloëdrische Krystalle der Combination $0 P, \infty P, \frac{1}{2} P$ denken will. Eine Gewissheit daruber, welche Annahme die richtige sei, konnte nicht erlangt werden, da die Versuche, deutliche Aetzfiguren auf der Basis herzustellen, scheiterten. Ich möchte mit Rücksicht auf das so häufig auftretende rhomboëdrische Aussehen der Krystalle, welches Scacchi auch an kunstlichen Krystallen von NatriumKaliumsulfat sehr deutlich - zugleich mit einer Verschiedenheit der FJächen 
von $+R$ und $-R-$ beobachtet bat (vergl. Zeitschr. d. d. geol.: Ges. 1865, 17, 41 unten), und da ich niemals eine unverkennbare $Z$ willingsbildung nach $0 R$ an den Douglashaller Krystallen gesehen habe, mich für das rhomboëdrische System entscheiden und annehmen, dass hier nur regelmässig verzerrte einfache Krystalle der Combination $0 R, \infty R, \frac{1}{2} R,-\frac{1}{2} R$ vorliegen.

Auffallend sind die den Aragonitdrillingen ähnlichen Krystalle des Natrium-Kaliumsulfats (Fig. 5 a und 5 b, 6 a und 6 b). Sie sind dicktafelartig nach $\{0001\} 0 R$ ausgebildet und zeigen randlich in der Regel nur $m=\infty R$, seltener auch noch $e=\frac{1}{2} R$ als schmale Abstumpfung der Kanten $(0 R, \infty R)$. Ueberaus bäufig lassen sie mebrfach sich wiederholende Einkerbungen in den Combinationskanten $c, m$ erkennen, hervorgebracht durch schmale Flächen der Rhomboëder $e$ und $e^{\prime}\left( \pm \frac{1}{2} R\right)$; doch habe ich niemals eine solche Regelmässigkeit gesehen, wie in Fig. 5 a und $5 \mathrm{~b}$ gezeichnet ist. Alle Krystalle dieser Ausbildung, an welchen vermöge ihrer Durchsichtigkeit eine opliscbe Untersuchung vorgenommen werden konnte, zeigten im convergenten Lichte $z$ wischen gekreuzten Nicols das Bild der optisch einaxigen Krystalle sehr deutlich; auch war dies der Fall bei einer aus einem ganzen Krystall hergestellten Platte nach $0 R$, welche bei einer schliesslichen Dicke von $1 \mathrm{~mm}$ an allen Stellen durchsichtig war.

Dagegen wurde an den Krystallen dieser Ausbildung nichts wahrgenommen von der Erscheinung, welche von Mallard (Bull. de la Sociélé minéral. de France 1882, 5, 226; vergl. auch diese Zeitschr. 9, 403) an Platten des "bexagonalen Kaliumsulfates (*), welche der Sammlung des Herrn Sénarmont zugehören, beobachtet wurde und welche darin besteht, dass die Platten zwar in der Mille nahezu einaxig sind, aber an dem Rande sich aus zweiaxigen Lamellen zusammensetzen, welche normal zu den Seitenflächen stehen und in der Ebene senkrecht zu der Combinationskante mit dem Prisma einen kleinen optischen Axenwinkel besitzen, - woraus dann Mallard schliessl, dass die scheinbare Einaxigkeit des mittleren Theiles der Platte eine Folge der Uebereinanderlagerung zweiaxiger Zwillingslamellen der gewöhnlichen rhombischen Form des Kaliumsulfats sei. Auch Erscheinungen, wie sie Wyrouboff (ebenda, 2, 100 etc.; vergl. diese Zeitschr. 4, 415) bespricht, habe ich nicht beobachtet.

Ich muss deshalb fưr das hexagonale System der den Aragonitdrillingen ähnlichen Krystalle eintreten und sie für einfache rhomboëdrische Krystalle erklären, bei welchen durch eine im Allgemeinen parallele Aneinanderlagerung, bezw. Fortwacbsung, einfacber rhomboëdrischer Krystalle

*) Zu vergl. ist Rammels berg, Supplement zum Handbuch der krystallograph. Chemie 1857, S. 34. - In der zweiten Auflage des Handbuches 1881, 1, 402, stellt R a mmels be r g offenbar dieselben Krystalle zum rhomboëdrischen "Natrium-Kaliumsulfat «. Ob eine Analyse vorliegt? - 
der Combination $0 R, \infty R, \pm \frac{1}{2} R$ an einzelnen Stellen einspringende Winkel zwischen den Flächen $\pm \frac{1}{2} R$ auftreten konnten. Dass es sich in der That um derartige Wachsthumserscheinungen handelt, folgt aus der Beschaffenheit des in Fig. 6 a und $6 \mathrm{~b}$ von oben und von unten abgebildeten Krystalles, bei welchem die Basis durchaus keine einheilliche, sondern eine vielfach gebrochene Fläche ist, die z. B. bei ${ }^{*}$ eine deutliche Vertiefung, von einer nach der Mitte des Krystalles hingewendelen Rhomboëderfläche $e^{\prime}$, einer Fläche $e$ und einer unregelmässig verlaufenden Rinne umschlossen, enthält. Allenfalls könnte man auch an Durchkreuzungszwillinge nach $0 R$ denken. Aetzfiguren, welche darüber hätten Aufschluss geben können, ob solche Zwillinge vorliegen, konnten an dem wenigen mir zur Verfügung stehenden Material nicht in deutlicher Weise erzielt werden. Auf keinen Fall möchte ich das Vorhandensein von solch' idealen Verwachsungen und von Zwillingsoder Drillingsbildungen von solch' ungemeiner Regelmässigkeit, wie sie Mallard und W y rouboff annehmen, voraussetzen.

An einigen der untersuchted Krystalle war eine ziemlich deutliche Spaltbarkeit nach $\infty R$ vorhanden, unter anderem auch an dem in Fig. 6 abgebildeten Krystalle, welcher in der angedeuteten Weise von einer Spaltungskluft parallel der vorderen $\infty$ R-Fläche durchsetzt wird. Dagegen kann die von G. v. Ha u e r (Journal für prakt. Chemie 1861, 83 (II), 357), allerdings wohl mehr mit Bezug auf das Ammoniumsulfat, und die von Scacchi (Zeitschr. d. d. geol. Ges. 1865, 1\%, 40) erwähnte "deulliche Spaltbarkeit nach $c$ « hier nur als eine sehr unvollkommene bezeichnet werden.

Der Charakter der Doppelbrechung wurde in allen von mir unlersuchten Platten als positiv erkannt, vollkommen in Uebereinslimmung mit S chra u f (vergl. Sitzber. d. Wien. Akad. 40, 598 und Journ. f. prakt. Ghem. 1861, 83 (II), 361) und Mallard (Bull. Soc. minér. 5, 226 und diese Zeitschr. 9, 403). Zur Bestimmung der Brechungsexponenten diente ein aus einem Krystall von rhombischem Aussehen hergestelltes Prisma, dessen brechende Kante der Verticalaxe annähernd parallel war. Der Winkel zwischen den mit Glasplättchen belegten Prismenflächen betrug $33^{0} 40^{\prime}$. Bei Anwendung von $\mathrm{Na}$-Licht war das Minimum der Ablenkung fur das ordentliche Bild des Websky'schen Spaltes $17029^{\prime}$, für das von dem ordentlichen sehr scharf getrennte ausserordentliche Bild des Spaltes $17048^{\prime}$, woraus hervorgeht

$$
\omega=1,4907 ; \quad \varepsilon=1,4993 .
$$

Diese Werthe zeigen eine grosse Uebereinstimmung mit den für das rhombische Kaliumsulfat von Topsoe und Christiansen (Pogg. Ann., Erg.-Bd. 6, 1874, 503 und 539) angegebenen Brechungsexponenten.

Bezüglich der chemischen Zusammensetzung der Krystalle bin ich in der angenehmen Lage, die durch die gütige Vermittelung des Herrn Consul Dr. Ochsenius in Marburg erhaltenen Angaben des Herrn A. Naupert 
in Westeregeln mittheilen zu können. Nach denselben hat Herr A. Geserick in Westeregeln drei ziemlich grosse Proben von den in Wasser leicht löslichen Krystallen untersucht und gefunden:

\begin{tabular}{|c|c|c|c|}
\hline & I. & II. & III. \\
\hline $\mathrm{K}_{2} \mathrm{SO}_{4}$ & 66,5 & 67,3 & 58,7 \\
\hline $\mathrm{Na}_{2} \mathrm{SO}_{4}$ & 22,0 & 18,2 & 19,5 \\
\hline $\mathrm{MgSO}_{4}$ & - & - & 3,4 \\
\hline $\mathrm{ClNa}$ & 10,1 & 11,6 & 14,4 \\
\hline Unlöslicbes & 0,4 & - & 0,1 \\
\hline Wasser u. Verlust & 1,0 & 2,9 & 3,9 \\
\hline & 100,0 & 100,0 & 100,0 \\
\hline
\end{tabular}

Die zu I. verwendelen Krystalle hatten eine etwas dunklere Farbe.

Das Chlornatrium ist, wie schon oben erwähnt wurde, als Einschluss in den Krystallen vorbanden. Man kann es auf den Bruchflächen der Krystalle an seiner guten Spaltbarkeit und dem starken Glanz der ebenen Spaltungsfläcben leicht erkennen. Es verräth sich namentlich auch bei dem Schleifen von Platten und Prismen an seiner uberaus leichten Löslichkeit; wo immer nur Einschlusse von Steinsalz beim Schleifen mit Wasser blossgelegı werden, entstehen sehr rasch unregelmässig die Krystalle durchziehende Höhlungen. Losgelöste Spaltungsstucke der stark glänzenden Einschlusse erwiesen sich bei weiterer Untersuchung als einfach brechend, von Würfelflächen begrenzt, und hatten, nach einer an $0,0270 \mathrm{~g}$ angestellten, von Herrn Dr. Linck ausgeführten Analyse, die Zusammensetzung $\mathrm{Cl}(\mathrm{Na}, \mathrm{K})$ mit ungefäbr $80 \% \mathrm{ClNa}$ und $20 \% \mathrm{Cl} \mathrm{K}$.

Man wird demnach die Krystalle obne Bedenken als schwefelsaures Natrium-Kalium betrachlen können, und zwar entsprechen die reineren, zu den Analysen I. und II. verwendeten Krystalle in ihrer Zusammensetzung ungefähr der Formel $5 \mathrm{~K}_{2} \mathrm{SO}_{4} \cdot 2 \mathrm{Na}_{2} \mathrm{SO}_{4}$, beziehungsweise $3 \mathrm{~K}_{2} \mathrm{SO}_{4} \cdot \mathrm{Na}_{2} \mathrm{SO}_{4}$, welche verlangt 75,4 \% $\mathrm{K}_{2} \mathrm{SO}_{4}$ und $24,6 \mathrm{Na}_{2} \mathrm{SO}_{4}$, beziehungsweise 78,6\% $\mathrm{K}_{2} \mathrm{SO}_{4}$ und $21,4 \% \mathrm{Na}_{2} \mathrm{SO}_{4}$. - Die Zusammensetzung $3 \mathrm{~K}_{2} \mathrm{SO}_{4} \cdot \mathrm{Na}_{2} \mathrm{SO}_{4}$ haben nach Penny (Journ. f. prakt. Chemie 1856, 67, 216) auch die zuerst von Mitscherlich untersuchten, aus der Kelplauge gewonnenen "platten " Natrium-Kaliumsulfatkrystalle. Ebenso besitzen die von S cacchi dargestellten, rbomboëdrischen Krystalle von Kaliumsulfat einen zwischen $15 \%$ und 24,5\% schwankenden Gehalt an schwefelsaurem Natrium (vergl. Zeilschr. d. d. geol. Ges. 1865, 1\%, 43).

In seiner Zusammensetzung steht somit das Mineral von Douglashall dem von Scacchi näher untersuchten Aphthalos vom Vesuv (Atti dell' Accad. delle scienze fisiche etc. 1873,5 , No. 3,29 und 1875,6 , No. 9, 48, an letzterem Orte auch Analysen) und dem von G. vom $R$ a th beschriebenen und von Diesem und von S a c chi (Neues Jahrh. f. Min. 1875, 620) analysirten 
Arcanit von Racalmuto sehr nahe. Mit dem ersteren Vorkommen hat es auch die Krystallform gemein. Ebenso sind die optischen Eigensehaften des Vesuvminerals vollkommen analog den an den Krystallen von Douglashall beobachteten; wenigstens konnte ich an dunntafelartig nach der Basis ausgebildeten Kryställchen und durchscheinenden Krusten von etwas grunlicher Farbe, welche angeblich im Jahre 1870 am Vesuv gesammelt wurden, im convergenten Lichte bei gekreuzten Nicols deutlich die Interferenzfigur der oplisch einaxigen Krystalle und den positiven Charakter der Doppelbrechung beobachten.

Bei der Aehnlichkeit der oben beschriebenen, an Aragonildrillinge erinnernden Krystalle mit den Arcanitkrystallen von Racalmuto dürfte ubrigens die von Scacchi im Jabre 1875 (Neues Jahrb. f. Min. S. 621, woselbst auch einige sehr naturgetreue Abbildungen der Krystalle von Racalmuto gegeben sind) ausgesprochene Vermuthung, dass der Arcanit G. vom R a th's rhomboëdrisch krystallisire, sehr an Wahrscheinlichkeit gewinne日, um so mehr als G. vo $\mathrm{m}$ R a th auch einige, allerdings sitzend ausgebildete Arcanilkrystalle von rhomboëdrischem Habilus (vergl. seine Fig. 27 in der citirten Abhandlung) an jener Stufe von Racalmuto beobachtet hat. Eine optische Untersuchung, welche am einfachsten in der noch offenen Frage uber das Krystallsystem des Arcanits von Racalmulo hätte Entscheidung bringen können, ist meines Wissens leider nicht ausgefuhrt worden. Sollte, was ich nicht für wabrscheinlich halten kann, das Mineral von Racalmuto doch, wie G. vom $R$ ath angenommen hat, rhombisch krystallisiren, oder sollte sich einmal irgendwo ein rhombisch krystallisirendes Natrium-Kaliumsulfat finden*), so möchte ich vorschlagen, fur das rbombische Mineral den von G. vom Rath fü dasselbe gebrauchten Namen Arcanit zu wählen. Das hexagonal krystallisirende Natrium-Kaliumsulfat wird man aber wohl am besten mit dem von Beudant (Traité de minéralogie, 2. éd. Paris, 2, 477) im Jahre $1832 \mathrm{ihm}$ gegebenen Namen Aphthalose, welchem gegenüber den anderen Namen die Priorität gebührt, oder mil dem in Deutschland und speciell in Douglashall mehr geläufigen, von $\mathrm{Hausmann}$ (Handbuch der Min. 1847, 2, 1137) herruhrenden Namen Glaserit bezeichnen.

*) Es ist wohl nicht überflüssig, darauf hinzuweisen; dass die Krystallformen, welche in vielen Lehrbüchern der Mineralogie für das rhombische, onit den Namen Aphthalit, Aphthitalit, Aphthalose, Arcanit und Glaserit bezeichnete Mineral angegeben werden, sich nicht auf natürliche, sondern auf künstliche Krystalle von Kaliumsulfat beziehen. Auch in Goldschmidt's Index der Krystallformen der Mineralien haben nur die von Brooke und Miller (Phillips, Mineralogy, London 1852, 534 etc.) angegeberien, an dem künsllichen Kaliumsulfat beobachteten Formen Berücksichtigung gefunden; aufallenderweise ist aber daselbst die Abhandlung G. vom Rath's über den Arcanit von Racalmuto ganz übersehen worden, ebenso dass $S$ cac chi in den dort $(2,86)$ doch citirten Arbeiten den Nachweis der rhomboëdrischen Natur des am Vesuv vorkommenden Glaserits (Aphthalos) erbracht hat. 


\section{Blödit (Astrakanit).}

In dem Astrakanitgesteine, welches die eben beschriebenen Glaseritkrystalle einschliesst, finden sich an einzelnen Stellen kleine, sehr stark glänzende Kryställchen, welche bei näherer Unsersuchung sich als dem Blödit (Astrakanit) zugehörig erwiesen. Das grösste und flächenreichste derselben, etwa $3 \mathrm{~mm}$ lang, wurde einer Messung unterzogen. Es ragt nur wenig aus dem Gesteine hervor, ist ausserdem nur von einer Seite ber ausgebildet, was fur die Identificirung von vornberein auf gewisse Schwierigkeiten stiess, und zeigt einen etwas anderen Habitus als die bisher von Stassfurt durch die Beschreibungen von G. vom Rath (Pogg. Ann. 144, 586), Groth und Hintze (Zeitschr. d. d. geol. Ges. 1871, 670 etc.) und L u d e ck e (Zeitschr. f. Naturwiss. Halle 1886, 69, 157 und diese Zeitschr. 13, 292) bekannt gewordenen Blöditkrystalle. Es wurden an dem in Fig. 7 in Projection auf eịe zur Prismenfläche senkrechte Ebene naturgetreu dargestellten Kryställchen folgende Flächen beobachtet :

(121) von $0=-2 R 2,(011)$ von $d=R \infty,(021)$ von $e=2 R \infty,(111)$ und (1T1) von $p=-P,(110)$ von $m=\infty P,(210)$ von $n=\infty P 2,(120)$ von $\nu=\infty R 2,(\overline{1} 11)$ von $u=+P,(\overline{2} 11)$ und $(\overline{2} \overline{1} 1)$ von $s=+2 \mathrm{P} 2 ;(\overline{2} 01)$ von $q=2 P \infty,(T 01)$ von $r=\mathrm{P}_{\infty}$.

Beim Abtrennen des Krystalles von seiner Unterlage; wobei ein Stuck mit den Flächen $s, q$ und $u$ absprang, kamen auf der Unterseite noch die drei Flächen $(\bar{T} 2 \bar{T})$ von $0,(\bar{T} T \bar{T})$ von $p$ und $(01 \bar{T})$ von $d$, zwar klein, aber sebr stark glänzend und eben, zum Vorschein. Auch war eine allerdings nicht sehr deutliche Spaltbarkeit nach $m$, die ich in der Literatur nicht erwäbnt finde, zu beobachten.

Eigenthumlich ist an dem Krystalle, dessen Flächen, wie bei allen bis jetzt bekannten Blöditkrystallen aus dem Stassfurler Salzlager, sebr gute Reflexbilder liefern, die verhăltnissmässig grosse Ausbildung der Flächen o, $d$ und $e$, das Fehlen, bezw. Zurticktieten der Formen $y\left\{\overline{2}_{21}\right\}$ und $\boldsymbol{x}\left\{\bar{T}_{21}\right\}$, und das Erscheheinen des primären Hemidomas $r$. Durch das Auftreten der letzteren Fläche näbern sich die Krystalle dem von $L$ u d e cke bescbriebenen Blödit von Stassfurt, unterscheiden sich aber von diesem durch die starke Entwicklung von $o\{121\}$, darin wieder mebr dem ersten von den anderen oben erwähnten Autoren beschriebenen Funde von Stassfurt ähnlich.

Die an dem Krystalle (Fig. 7) gemessenen Winkel sind in der folgenden Tabelle aufgezäblt; sie zeigen mit den Winkeln, welche sich aus den von Groth und Hintze aufgestellten Elementen berechnen und zum Vergleiche in der zweiten Spalte genannt sind, eine sehr gute Uebereinstimmung. Die genaueren, an sehr gut spiegelnden Flächen gewonuenen Werthe sind durch ein angefugtes $(a)$ von den weniger guten $(b)$ und den schlechteren (c) unterschieden. 


\begin{tabular}{|c|c|c|}
\hline & Gemessen : & Berechnet \\
\hline$d: 0=(0.11):(121)=$ & $23^{0} \quad 4^{\prime}(a)$ & $23^{0} 1^{\prime}$ \\
\hline$d: e=(011):(021)=$ & $1928 \quad(a)$ & 1926 \\
\hline$e: 0=(011):(121)=$ & $16 \quad 3 \frac{1}{2}(a)$ & 163 \\
\hline$p: 0=(111:):(121)=$ & $1857 \quad(b)$ & 1853 \\
\hline$p: d=(1 \mid 1):(011)=$ & $21 \quad 1 \frac{1}{2}(a)$ & 214 \\
\hline$p: p=(111):(1 T 1)=$ & $5732 \quad(b)$ & $57 \quad 42 \frac{1}{2}$ \\
\hline $0: 0=(121):(T 2 \pi)=$ & $8432 \cdot(b)$ & 8431 \\
\hline $\begin{aligned} o: m= & (121):(110)= \\
& (12 \pi):(110)=\end{aligned}$ & $\left.\begin{array}{lll}34 & 38 \frac{1}{2} & (a) \\
34 & 44 & (b)\end{array}\right\}$ & 3449 \\
\hline$d: m=(011):(110)=$ & $57 \quad 42 \frac{1}{2}(a)$ & 5750 \\
\hline$r: m=(\overline{1} 01):(011)=$ & $10014 \frac{1}{2}(b)$ & 10030 \\
\hline$s: m=(\overline{2} T 1):(011)=$ & $13551 \quad(c)$ & 1359 \\
\hline$e: m=(021):(011)=$ & $4511 \quad(a)$ & $45 \quad 17 \frac{1}{2}$ \\
\hline$e: u=(021):(T+1)=$ & $2837 \frac{1}{2}(b)$ & 2756 \\
\hline$e: q=(021):(\overline{2} 01)=$ & $6716 \frac{1}{2}(b)$ & 6710 \\
\hline$d: u=(011):(\bar{T} 11)=$ & $2342 \frac{1}{2}(b)$ & 2334 \\
\hline$u: s=(\bar{T}: 11):(\overline{2}, 11)=$ & $1934 \quad(c)$ & 1913 \\
\hline$s: q=(\mathrm{T} 11):(\overline{2} 01)=$ & $2732 \quad(b)$ & 2725 \\
\hline$d: q=(011):(\overline{2} 01)=$ & $57 \quad 46 \quad(b)$ & 57.37 \\
\hline$d \because n \doteq(011):(2,0)=$ & $\begin{array}{llll}64 & 17 & (b)\end{array}$ & 6436 \\
\hline$m: n=(110):(210)=$ & $1931 \frac{1}{2}(b)$ & 1927 \\
\hline$\nu: m=(120):(110)=$ & $\begin{array}{lll}36 & 23 & (c)\end{array}$ & 3621 \\
\hline
\end{tabular}

Yergl. auch die Nachscbrift am Schlusșe, Beite 575.

\section{Kainit.}

Unter den von den Herren Dr. Ochsenius und A. Naupert mit dankenswertber Bereitwilligkeit zur Bearbeitung ithersendeten Mineralien von Douglashall entdeckte ich auch Krystalle von Kainit. Dieselben scheinen dort sebr selten zu sein. Sie finden sich in einem in der Kieseritregion erschlossenen Lager von derbem Kainit als kleine, elwa 3 bis $4 \mathrm{~mm}$ grosse, farblose bis schwach gelb gefärbte, durchsichtige Kryställchen, welche in Drusen dicht gedrängt neben einander sitzen, von der Seite her aufgewachsen und nur wenig aus der Unterlage, dem derben Mineral, hervorragend. Entweder sind sie durch Vorwalten der Basis tafelartig oder durch grössere Entwickelung der primăren Hemipyramiden $o$ und $o^{\prime}$ flach pyramidal ausgebildet (vgl. Fig. 8, welche einen Krystall in Projection auf eine Fläche 
senkrecht zur Prismenzone darstellt). Alle anderen beobachteten naturlichen Flächen erscheinen an dem untersuchten Krystall nur klein; besonders gilt dies von der Symmetrieebene $b$. Das Orthopinakoid $a$ tritt nicht als natürliche, sondern nur als Spaltungsfläche auf.

Die Spaltung nach $a$ ist eine sehr vollkommene und kann auch allenthalben an den derben Stucken beobachtet werden. Eine weitere, zwar weniger vollkommene, aber doch immerhin noch recht deutliche Spaltbarkeit geht nach den Flächen der Hemipyramide o(111); nur unvollkommen, auch nur einmal beobachtet, ist eine drilte Spaltbarkeit nach der Hemipyramide $o^{\prime}(111)$. Die prismatische Spaltbarkeit (nach $p(110)$ ) wurde nicht wahrgenommen.

Die an dem gemessenen Krystalle bestimmten Formen sind folgende:

$$
\begin{gathered}
c\{001\} 0 P, \quad b\{010\} \infty R \infty, \quad a\{100\} \infty P \infty, \quad 0\{111\}-P, o^{\prime}\{111\}+P, \\
r\{201\}-2 P \infty, p\{110\} \infty P .
\end{gathered}
$$

Unter diesen zeichnen sich $a, c, o^{\prime}$ und o durch vollkommen ebene, stark glänzende Flächen aus, so dass die Winkel zwischen denselben mit grosser Genauigkeit - in der Tabelle durch ein beigefugtes $(a)$ angedeutet - bestimmt werden konnten. Weniger gut ausgebildet $(b)$ sind die Formen $r, p$ und $b ; r$ giebt einen verschwommenen Reflex, $p$ ist in der Richtung der Prismenzone elwas gekrummt, und $b$ ist eine sehr kleine, das Licht nur sebr schwach reflectirende Fläche. Nicht sicher bestimmbar $(c)$ ist ein Prisma $\pi$, welches zwischen $p$ und $a$ gelegen, in dieser Richtung stark gekrümmt erscheint, und eine negative Brachypyramide $\xi$, welche in der Zone $a, o^{\prime}, p$ gelegen, wegen der drusigen und gekrümmten Beschaffenheit ihrer Fläche nur sehr unvollkommen spiegelt. Das Prisma $\pi$ hat annähernd die Lage, welche ein Prisma $\{980\} \infty P_{\frac{9}{8}}^{\circ}$ besitzen wurde, die Brachypyramide $\xi$ hat die Lage von $\{4.18 .7\}-\frac{18}{7} R \frac{9}{2}$, isl aber vielleicht auf die äbnlich gelegene von V. von $Z$ epharovich beobachtele Hemipyramide $-3 \boldsymbol{R 3}$ \{131\} zu beziehen. Der Vollständigkeit halber sind auch die für diese Flä-

\begin{tabular}{|c|c|c|}
\hline Gemessen: & Berechnet: & $\begin{array}{l}\text { Berechnet von Groth } \\
\text { u. v. Zepbarovich: }\end{array}$ \\
\hline$a(100): c(001)={ }^{*} 85^{0} \quad 0^{\prime}(a)$ & - & $85^{0} \quad 5,5$ \\
\hline$o^{\prime}(\bar{T} T 1): a(\bar{T} 00)=* 71 \quad 5(a)$ & - & - \\
\hline$o^{\prime}(\bar{T} T 1): c(001)={ }^{*} 38 \quad 18 \quad(a)$ & - & $38 \quad 14,5$ \\
\hline$o^{\prime}(T T \Lambda): o(1 T 1)=45 \quad 10(a)$ & $45^{0} 12^{\prime}$ & - \\
\hline$o(1 T 1): c(001)=3558(a)$ & $35 \quad 59$ & $35 \quad 58$ \\
\hline$o(4 \pi 1): a(100)=6345(a)$ & 6343 & - \\
\hline 10$)=63 \quad 10(b)$ & 632 & 6259,5 \\
\hline$p\left(1 T 0_{i}: o(1 T\}\right)=5055(b)$ & 5050 & 5054,5 \\
\hline
\end{tabular}
chen erhaltenen Winkel in der folgenden Tabelle mit aufgefuhrt. 


\begin{tabular}{|c|c|c|}
\hline Gemessen : & Berechnet: & $\begin{array}{l}\text { Berechnet von Groth } \\
\text { u. v. Zepbarovich: }\end{array}$ \\
\hline$p(1 \bar{\top} 0): c(001)=86^{0} 53^{\prime}(b)$ & $86^{0} 49^{\prime}$ & $86^{\circ} 53^{\prime}$ \\
\hline$p(\bar{T} \bar{T} 0): o^{\prime}(\bar{T} \bar{T})=54 \quad 43(b)$ & 5453 & 5452,5 \\
\hline$p(1 \overline{1} 0): a(100)=5024(b)$ & 5024 & 5031,5 \\
\hline$r(201): a(100)=4335(b)$ & 43. 25 & 4333,5 \\
\hline$r(201): c(001)=4125(b)$ & 4135 & 4132 \\
\hline$r(201): o(1 \overline{1})=3116(b)$ & 3126 & - \\
\hline$\pi(9 \overline{8} 0): a(100)=46 \quad 46 \quad(c)$ & 474 & - \\
\hline$\xi(4 . \overline{1} \overline{8} . \overline{7}): o^{\prime}(\bar{T} T 1)=3935$ (c) & 3944 & - \\
\hline
\end{tabular}

Aus den mit * bezeichneten, sebr genau ermittelten Werthen berechnet sich das Axenverhältniss

$$
\begin{gathered}
a: b: c=1,2138: 1: 0,5862 \\
\beta=85^{0} 0^{\prime},
\end{gathered}
$$

welches nur wenig von dem von Grolh angegebenen, auch von V. v. Zepharovich (diese Zeilschr. 6, 234 etc.) adoptirten,

$$
a: b: c=1,2186: 1: 0,58631 ; \beta=85^{0} 5 \frac{1}{2}^{\prime}
$$

abweicht. Welches von heiden für die Krystalle von Douglashall vorzuziehen ist, dürften erst Untersuchungen an umfangreicherem Material entseheiden können.

Die optische Untersuchung ergab Werthe, welche mit den von V. von Zepharovich a. a. 0 . milgetheilten eine im Allgemeinen recht gute Uebereinstimmung zeigen, aber bei der ungeeigneten Beschaffenheit des zur Verfugung stehenden Materials und der dadurch bedingten Schwierigkeit, genau orientirle Platten herzustellen, doch keinen Anspruch auf sebr grosse Genauigkeit machen können.

Man siebt durch Spaltungsblättchen nach $a(100)$ bei Anwendung von $\mathrm{Na}$-Licht im Polarisationsinstrumente den mittleren Theil des Lemniscalensystems fast ganz in der Mitte des Gesichtsfeldes und kann an solchen Blättchen den in der Symmetrieebene gelegenen optischen Axenwinkel, welcher in Luft nicht beslimmbar ist, in Oel oder Alphamonobromnaphthalin messen. Für $N a$-Licht beträgt der scheinbare Axenwinkel in Oel 970 52', in Brom= naphtalin $83^{\circ} 52^{\prime}$.

Durch $c(001)$ ist eine Axe im stumpfen Winkel $\beta$, etwa neun Theilstriche von der Mitte der Mikrometertheilung entfernt, sichtbar, während das Centrum des Lemniscatensystems nach der anderen Seite hin, also im spitzen Winkel $\beta$, etwa vier Theilstriche von der Mitte der Mikrometertheilung entfernt, gelegen ist. Es entspricht das ungefähr der von V. von Zepharovich und Groth gemachten Angabe; dass die eine Elasticilätsaxe mit der $c-A$ xe im spitzen Winkel $\beta$ einen Winkel von $10^{0} 43^{\prime}$ bis $8^{0}$ bildet. 
Krystallbruchstucke und optisch geprifte Spaltungsstücke wurden von Herrn Dr. Linck einer chemischen Analyse unterzogen. Die mit zwei Proben von 0,2091 bezw. 0,5392 g Gewicht erhaltenen Resultate sind die in der folgenden Tabelle unter 1 . und 2 . aufgefuhrten.

\begin{tabular}{lccc} 
& 1. & 2. & 3. \\
$\mathrm{Cl}$ & 13,97 & - & 14,29 \\
$\mathrm{~K}$ & 14,99 & - & 15,70 \\
$\mathrm{MgO}$ & 16,61 & - & 16,10 \\
$\mathrm{SO}_{3}$ & 34,30 & - & $: 32,19$ \\
$\mathrm{H}_{2} \mathrm{O}$ & - & 21,11 & 21,72 \\
\cline { 2 - 3 } & \multicolumn{2}{c}{100,98} &
\end{tabular}

Die Uebereinstimmung der gefundenen Werthe mit der von anderen Analytiker'n für den Kainit angegebenen ist eine recht gute. Die aus der Formel $\mathrm{MgSO}_{4} . \mathrm{KCl} .3 \mathrm{H}_{2} \mathrm{O}$ berechneted Werthe (vgl. Rammelsberg, Mineralchemie) sind zum Vergleich in der dritten Columne hinzugefugt.

\section{Boracit.}

Krystalle von Boracit finden sich zu Douglashall vereinzelt und von geringer Grösse, höchstens bis $1 \mathrm{~mm}$ gross, ganz regelmässig im Carnallit, und zwar, wie es scheint, besonders in den jügeren Schichten desselben. In grösserer Häufigkeit und zum Theil von ansehnlicher Grösse sind sie, wie ich einer freundlichen Mittheilung der Herren Ochsenius und.Naupert entnehme, namentlich in zwei Perioden vorgekommen, nämlich im Juli bis September 1885, wo etwas gelbliche, aber durchsichtige Kryställchen gefunden wurden, und dann später von November 1887 bis Februar 1888 , zu welcher Zeit sich prachtvolle, bis $3 \mathrm{~mm}$ grosse, wasserhelle und bis $5 \mathrm{~mm}$ grosse, lichtgrunlich gefärbte durchsichtige Krystalle einstellten.

Krystalle dieses letzten Fundes sind durch die Vermittelung der Mineralienbandlung von Kran $\mathrm{z}$ in Bonn. in viele Sammlungen gelangt. Sie erscheinen entweder als. Würfel, an welchen das Rhombendodekaëder nur ganz schmal die Kanten abstumpfend auftritı oder als. Combinationen des Würels mit dem ziemlich gross entwickelten Tetraëder und dem bald schmälere, bald breitere Flächen zeigenden Rhombendodekaëder. Bei den grün gefärbten, besonders bei Lampenlicht prachtvoll aussehenden Krystallen ist das Tetraëder häufig uber den Würfel vorherrschend; auch Tetraëder, an welchen der Würfel nur als ganz schmale Kantenabstumpfung erscheint, sind nicht selten vorgekommen. Ein durchgreifender Unterschied in der Ausbildung ist aber zwischen den grünen und wasserhellen Boracitkrystallen nicht vorhanden.

Nur vereinzelte unter etwa $1500 \mathrm{Krystallen,} \mathrm{welche} \mathrm{mir,} \mathrm{dank} \mathrm{der}$ Liebenswurdigkeit des Herrn A: Na pert zur Durchsicht vorlagen, sind 
flächenreicher ausgebildet. So zeigt ein gruner Krystall (Nr. 2), bei vorherrschendem Wurfel und mehr zurucktretendem Tetraëder und Rhombendodekaëder, auch noch ganz schmal den von $\mathrm{Schrauf}$ (Atlas der Krystallformen, 1873, Taf. 36) zuerst an den Stassfurter Boraciten beobachteten Pyramidenwürfel $i\{013\} \propto 03$, an einer Würfelkante deutlich mit zwei Flächen entwickelt. Der Winkel (100):(310) wurde bestimmt zu 1808'; der berechnete ist $18^{\circ} 26^{\prime}$.

Ein zweiter grüner Krystall (Nr. 1) ist ähnlich beschaffen; $a\{001\}$ $\infty 0 \infty$ ist vorherrschend, $x\{111\}$ o und $d\{011\} \infty 0$ treten mehr zuruck; von $d$ sind einzelne Flächen grösser als die übrigen (vgl. Fig. 9, welche den Krystall auf diejenige Würfelfläche projicirt zeigt, auf welcher er am flächenreichslen ausgebildet ist). Mit etwas breiteren Flächen erscheint der Pyramidenwürfel $\pi=\{014\} \infty 04$, auch mit der Würfelfläche $a$ mehrfach alternirend, wodurch auf dieser eine doppelte Streifung, nach beiden Würelkanten, entsteht. Der Winkel (001):(041) wurde, bei besserer Flächenbeschaffenheil von $\pi$, zu 14.022', bei etwas schlechterer Beschaffenheit zu $130{ }^{\circ} 7^{\prime}$ bestimmt; der berechnete ist $14^{0} 2^{\prime}$. Ferner wurden an dem von einzelnen Rinnen durchfurchten, offenbar mebrfach in seinem Wachsthum unterbrochenem Krystall auf derselben Wurfelfäche, aber nur in e in er Zone, schmale, mit der Würfelfläche alternirende Flächen eines zweiten Pyramidenwürfels aufgefunden, welche einen deutlichen Reflex lieferten und mil der Fläche $a$ den Winkel $5^{0} 5^{\prime}$ einschliessen; daraus würde auf das Zeichen $\infty 011\{0.1 .11\}$ (ber. $5^{0} 12^{\prime}$ ) oder auf das Zeichen $\infty 012\{0.1 .12\}$ (ber. $4^{0} 46^{\prime}$ ) geschlossen werden können, von welchen jedenfalls das letztere den Vorzug verdient. Ganz schmal und etwas gerundet sind Flächen eines Pyramidenwürfels, welche mit den Wüfelflächen die nur approximativ zu bestimmenden Winkel $18^{\circ} 6^{\prime}, 19^{0} 13^{\prime}, 19^{\circ} 40^{\prime}, 20^{\circ} 38^{\prime}$ und $20^{\circ} 41^{\prime}$ bilden, also Winkel, wie sie $\{013\} \infty 03$ und $\{025\} \infty 0$ 音 (ber. 18026', bezw. 21048'), oder $\{0.4 .11\} \infty 0^{11}{ }^{1}$ (ber. $\left.19^{\circ} 5^{\prime} 9^{\prime}\right)$ zukommen würden; wahrscheinlich liegt hier die von Schrauf schon im Jahre 1873 beslimmte, auch an dem vorher erwähnten Krystalle vorhandene Form $\{013\} \infty 03$ vor.

Ein dritter Krystall (Nr. 3), ebenfalls blessgrün, zeigt ausser dem vorherrschenden Würfel ziemlich gross entwickelt das Tetraëder, schmale Flächen des Rhombendodekaëders, dann aber noch eine feine Abstumpfung der Kanten zwischen Würfel und Tetraëder durch $x\{211\} n^{\prime}$. Der Winkel (211): (100) beträgt nach einer approximativen Bestimmung 36017' der berechnete ist $35^{0} 16^{\prime}$.

Ein gruner Krystall (Nr. 5) ist von tetraëdrischem Habitus durch Vorwalten von $x\{111\} O$; ziemlich gross sind die Wurfelflächen; auch "die Dodekadërflächen; mit kleinen, aber sehr stark glänzenden, gut spiegelnden Flächen sind $x\{\bar{Q} \bar{T} T\} n$ und $x\{\bar{T} \bar{T}\} o^{\prime}$ ausgebildet; auch Andeutungen eines Pyramidenwurfels und eines positiven Triakistetraëders sind vorhanden. 
Ganz besonders interessant ist noch ein wasserheller Krystall (Nr. 4) von $3 \mathrm{~mm}$ Durchmesser (Fig. 10a und b), bei welchem $a$ und $o$ im Gleichgewicht entwickelt sind. Seine Flächen sind äusserst eben und geben fast sämmtlich scharfe deutliche Reflexbilder. Ausser $0=x\{111\}$ und $a\{001\}$ erscheint an ihm noch $d\{110\}, x\{\bar{T} \bar{T}\} o^{\prime}, x\{\overline{2} \bar{T} \bar{T}\} n$, ferner zwar sehr klein, aber genau beslimmbar $x\{144\} \varrho$ als Abstumpfung der Combinationskanten od, sowie als ganz schmale Abstumpfung der Kanten $o^{\prime} d$, bezw. als Streifung auf $d$ parallel der Kante $o^{\prime} d, x\{(\overline{1} \overline{8} \overline{8}\} \sigma$ und $x\{\bar{T} \cdot \overline{1} \overline{6} \cdot \overline{1} \overline{6}\} \tau$. Die recht genau messbaren Winkel $(441):(110),(8 \overline{8} 1):(110),(16.1 \overline{6} .1):(110)$ wurden bestimmt zu $10^{\circ} 2^{\prime}$, bezw. $4^{0} 59^{\prime}, 2^{0} 14^{\prime}$, während die berechneten Werthe $10^{0} \frac{1}{2}^{\prime}$, bezw. $5^{0} 3^{\prime}, 2^{0} 32^{\prime}$ betragen. Nur annäher'nde Messungen erlaubt ein $+x\{11 m\}$, wahrscheinlich $x\{112\}$, und ein $x\{\overline{1} \bar{m}) \xi$. Fur das letztere bestimmte sich der Winkel $(1 T m):(1 T 0)$ auf 12034', während er für $m=6$ sich auf $13^{0} 16^{\prime}$, fur $m=\frac{13}{2}$ auf $12017^{\prime}$ berechnel.

Die von $\mathrm{Sch}$ rauf beobachtete Form $\Sigma=\chi\{255\}$ habe ich an den von mir gepruften Krystallen nicht aufgefunden.

An den Boracilkrystallen aus dem Carnallit des Stassfurter Steinsalzlagers sind demnach zur Zeit folgende Formen bekannt.

1) An den Krystallen von Stassfurt, durch Schrauf nachgewiesen: $a\{001\}, d\{011\}, i\{013\}, o=x\{111\}, \mathbf{\Sigma}=x\{255\}, n=x\{\pi 12\}$.

2) An den Krystallen von Westeregeln :

$$
\begin{gathered}
a\{001\}, d\{011), i\{013\},{ }^{*} \pi\{014\}, o=x\{111\}, n^{\prime}=x\{112\},{ }^{*} Q=x\{14.4\}, \\
o^{\prime}=x\{\overline{1} 11\}, n=x\{112\},{ }^{*} \sigma=x\{188\},{ }^{*} \tau=x\{1.16 .16\} .
\end{gathered}
$$

Noch nicht genugend sicher gestellt sind die Formen $\{0.1 .12\}$ und $\xi=$ \{116\}. Die mil einem * bezeichnelen Formen sind bisher am Boracil noch nichl beobachtet worden*).

Die bald hellere, bald dunklere Farbe der grunen Boracite, welche im Allgemeinen viel seltener sind, als die wasserhellen, ist bedingt durch einen Gehalt an Eisenoxydul. Bei der Analyse von $4 \mathrm{~g}$ der dunkleren Varielät fand Herr Dr. Wense aus Luneburg, wie Herr A. Na upert gütigst mittheilt, 7,9\% $\mathrm{FeO}$.

Unter den mir vorliegenden Boraciten sind auch einzelne, durch ungleichmässig aufgelagerle kleine Eisenglanzscbüppchen rothgefärbte, bezw. roth gefleckte, ferner schwach gelbgefärbte und weisse, durch zahlreiche Interpositionen winziger unregelmässig aufgewachsener Kryställchen, anscheinend von Boracit, trube undurchsichtige Krystalle. Ausser den einzelnen, ringsum ausgebildeten Krystallen sind auch Gruppen von mehreren, zuweilen bis 20 , unregelmässig mit einander verwachsenen Krystallen

*) $n^{\prime}=x\{112\}$ ist von Goldschmidt in seinem Index der Krystallformen nicht aufgeführt, wird aber von Miller, Min. 1852, S. 602 angegeben. Jedenfalls ist die Form recht sellen. 
vorhanden. Die von $S c h r a u f$ erwähnte $Z$ willingsverwachsung habe ich an dem mir vorliegenden Material nicht bemerkt.

Kleine Knollen von hartem dichtem Boracit (sog. Stassfurtit), von Stecknadelkopf- bis Haselnussgrösse, finden sich auch zu Douglashall im Carnallit ganz ebenso wie bei Stassfurt.

Strassburg, 9. März 1889.

Nachschrift. Inzwischen habe ich durch gulige Vermittelung des Herrn A. Naupert in Douglashall auch einige grössere Blöditkrystalle erhalten, welche sich vor Kurzem in mit Lauge angefüllten Drusen des oben erwähnten Astrakanitgesteines gefunden haben. Diese Krystalle sind durchschnittlich $50 \mathrm{~mm}$ lang, $40 \mathrm{~mm}$ breit und $20 \mathrm{~mm}$ dick, weisen aber zum Theil auch noch grössere Dimensionen auf. Sie sind gewöhnlich von der Seite aufgewachsen und bisweilen modellartig ausgebildel. Kurzprismaliscb, bezw. dicktafelartig nach $c$ entwickelt, zeigen sie in der Prismenzone vorherrschend die Formen $m\{110\}$ und $n\{210\}$, klein $\nu\{120\}$ und $b\{010\}$, an beiden Enden gross die Flächen $c\{001\}$ und $p\{111\}$, etwas kleiner $d\{011\}$ und $0\{121\}$, ganz klein $e\{021\}$. Die ausgezeichnete Flächenbeschaffenheit dieser Krystalle wird es ermöglicben, die krystallographiscben und optischen Verhältnisse des Douglashaller Blödits mit der gleichen Genauigkeit zu prüfen, wie dies für das Stassfurter Vorkommen durch Groth und Hintze geschehen ist. 